\title{
ACEPTACIÓN Y ADOPCIÓN DE SISTEMAS PRODUCTIVOS ADAPTATIVOS EN LA MICROCUENCA EL GUAYABO-SAN JOSÉ, MUNICIPIO EL SAUCE, LEÓN
}

\section{ACCEPTABILITY AND ADOPTION OF ADAPTATIVE PRODUCTION SYSTEMS IN THE WATERSHED “EL GUAYABO-SAN JOSÉ”, EL SAUCE MUNICIPALITY, LEON}

Toruño Pedro José1, Roque-Gómez Johanna Lissette, Torres-Ríos Zeneyda Antonia

${ }^{1}$ Docente e investigador en Agroforestería y Manejo de Cuencas, Universidad Nacional Autónoma de Nicaragua, León E-mail: pjoseto@unanleon.edu.ni

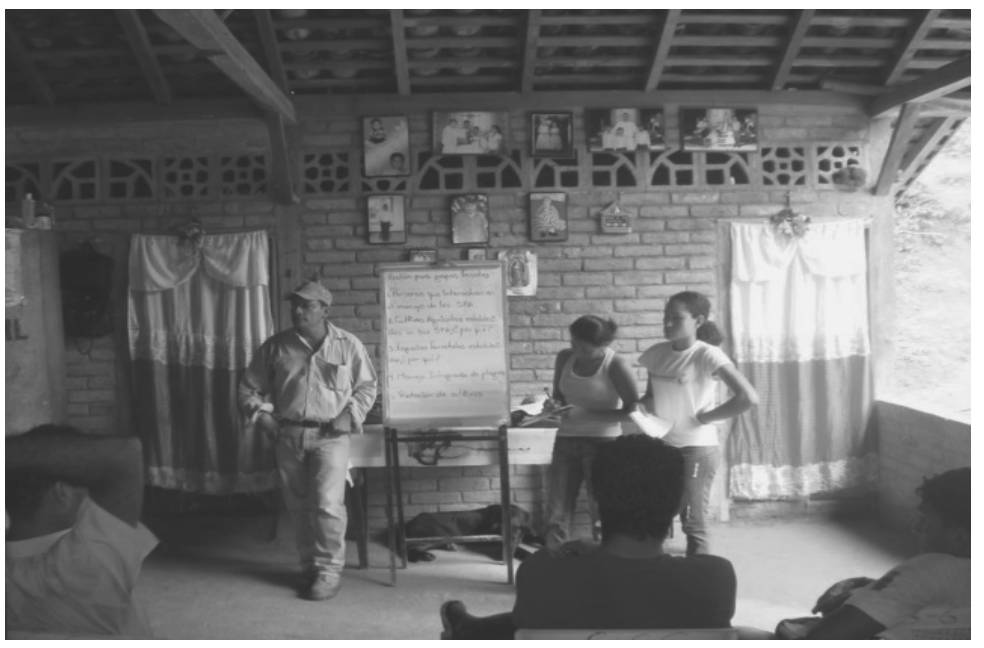

\section{RESUMEN}

El establecimiento de Sistemas Productivos Adaptativos (SPA), constituye una alternativa de producción sostenible y compatible con la conservación del medio ambiente; sin embargo se desconocen los elementos que inciden en los proceso de aceptación y adopción. El objetivo de esta investigación es analizar desde la perspectiva social la aceptación, adopción e integración de género en los Sistemas Productivos Adaptativos en la microcuenca "Guayabo-San José". Los SPA estudiados fueron granos básicos en callejones mejorados y granos básicos con árboles dispersos. El presente es un estudio cualitativo, la información se obtuvo a través de triangulación de métodos: entrevistas, observación participante y grupos focales. Se realizó un análisis de contenido obteniéndose como resultados una percepción positiva de aceptación por parte de los productores. Existe un $100 \%$ de aceptación y un $25 \%$ de adopción de los sistemas.La adopción aumenta cuando los productores disponen de tierra propia, beneficios extras en sus parcelas y adecuadas capacitaciones y asistencia técnica. Sobre el rol que las cónyuges desempeñan, las entidades no tomaron en cuenta su involucramiento en el proceso de transferencia de los sistemas. Las cónyuges no se involucran de manera directa en el manejo de los sistemas, desempeñando únicamente actividades domésticas y el cuido de los hijos. Los productores no realizan todas las prácticas de manejo del sistema; sin embargo, se incluyen los tres componentes del modelo productivo.

Palabras clave: Sistemas productivos adaptativos, género, aceptación, adopción.

Abreviaturas: CSA, Conservación de suelo y Agua, GBAD, Granos Básicos con Árboles Dispersos, GBCM, Granos Básicos en Callejones Mejorados, MST, Manejo Sostenible de la Tierra, SAF, Sistemas Agroforestales, SPA, Sistemas Productivos Adaptativos.

\section{ABSTRACT}

Establishing Adaptive Production Systems (SPA) is an alternative to support sustainable production and conservation of the environment. However, factors influencing the acceptance and adoption process of these production systems are unknown. The objective of this research was to analyze social perspective acceptance, adoption and integration of gender in the Adaptive production systems in the watershed "Guayabo-San José". The SPA studied were basic grains in improved alley and basic grains planted with scattered trees. This is a qualitative study; information was obtained through triangulation of methods: interviews, participant observation and focus groups. We conducted a content analysis obtaining as a result a positive perception of acceptance by producers. There is a $100 \%$ acceptance and 25\% adoption of Adaptive Production Systems. The adoption increases when farmers have land of their own, extra benefits in their land and appropriate training and technical assistance. On the role that spouses play, the organizations did not consider for their involvement in the transfer process systems. The spouses are not involved directly in the management of systems, doing only household tasks and the care of children. Farmers do not carry out all system management practices, but include all three components of the production model.

Keywords: adaptive production systems, gender, acceptance, adoption.

Abbreviations: CSA, Soil and Water Conservation, GBAD, basic grains with scattered trees, GBCM, enhanced Basic Grains in alleys, MST, Sustainable Land Management, SAF, Agroforestry, SPA, Adaptive Production Systems. 
$\mathrm{E}$ n el último medio siglo la población nicaragüense ha presentado un importante crecimiento poblacional, en donde la agricultura y la ganadería representan una fuente esencial de ingresos para la economía doméstica; Sin embargo, la producción agrícola, ganadera y forestal se ha llevado a cabo con sistemas productivos de bajos rendimientos que deterioran el medio ambiente, debilitando el equilibrio de los ecosistemas, la productividad de la tierra, la disponibilidad de agua, la riqueza de la biodiversidad y los bosques.

El municipio El Sauce no escapa a esta situación, en donde las actividades productivas están en manos de pequeños productores. En su mayor parte se realizan en laderas, de forma tradicional (provocado una enorme presión en nuestros recursos naturales) y con bajos rendimientos (Arauz, 2010). Por lo cual se hizo necesaria la identificación y promoción de nuevas prácticas productivas sostenibles, que eviten la destrucción o degradación del medio ambiente, siendo económica y financieramente viables, socialmente pertinentes y técnicamente aceptables (Barzev, 2004).

En busca de una solución en los años 80 la UNAG crea el Programa "De campesino a campesino" creando un proceso de transformación a una agricultura con bajas cargas de insumos químicos. En los años 90 el Proyecto Manuel López inicia un proceso de transformación humana cuyo propósito era el Desarrollo Rural Integral (Osorio, 2010).

En el año 2006 en ejecución del MARENA se inicia el Proyecto Manejo Sostenible de la Tierra (MST), partiendo de un diagnóstico previo, que reveló que más del $80 \%$ de la producción agrícola se basa en granos básicos y la existencia de un excelente potencial hídrico (Cáceres, 2010).

Evidenció también que la producción agrícola, ganadera y forestal se lleva a cabo con sistemas productivos de bajo rendimientos y de gran impacto negativo al medio ambiente, así mismo la vulnerabilidad de los recursos naturales. Para superar esta situación el proyecto propuso el establecimiento de modelos productivos como: Agroforestales (GBAD y GBCM), Silvopastoril (Laderas arbustivas) y Forestales (Protección de Ojos de agua, Bosques de Galerías y Manejo forestal sostenido en Bosque Seco Latifoliado), orientados a revertir la problemática ambiental, particularmente el deterioro de la tierra, y a incrementar sustancialmente la producción agropecuaria y forestal (Manejo Sostenible de la Tierra et al., 2005).

Aunque el establecimiento de los Sistemas Productivos Adaptativos (SPA) constituye una alternativa de producción sostenible y compatible con la conservación del medio ambiente, es importante mencionar que en El Sauce se carece de trabajos de investigación que permitan conocer con seguridad el impacto social de los proyectos que se han implementado en los últimos 30 años, lo que dificulta el proceso de desarrollo y sostenibilidad de los mismos, impidiendo mejorar sus estrategias de transferencia, ya que se desconocen los elementos que inciden en este proceso considerándose esta una línea de investigación casi inexistente en las estructuras institucionales.

En tal sentido el propósito de esta investigación es describir la percepción de los productores sobre la aceptación y adopción de los SPA, conocer su manejo implementado por los productores y señalar el rol de género (las mujeres cónyuges de productores) en la implementación de los SPA en la microcuenca "El Guayabo-San José". Y de esta manera poner a disposición información sobre los aspectos relacionados a aceptación y adopción desde la perspectiva social de los SPA establecidos por los productores, lo cual permitirá que otras entidades del sector agropecuario y forestal cuenten con elementos útiles para la toma de decisiones de nuevas inversiones, ya sea a nivel de proyectos de desarrollo rural o de investigación para el desarrollo.

\section{MATERIALES Y MÉTODOS}

Ubicación del estudio. El área de estudio fue la microcuenca "Guayabo-San José", ubicada a $12 \mathrm{~km}$ al sureste de la cabecera municipal de El Sauce, con un área aproximada de $70 \mathrm{Km}^{2}$, abarca cuatro comarcas (Sabana Grande, Las Mercedes, Ocotal y Los Tololos), representativas del municipio en aspectos de población, clima, suelo y sistemas de producción. Del total de la población, el $25 \%$ viven en la parte alta de la cuenca; el $30 \%$ en la parte media y el 45 $\%$ habitan en la parte baja donde hay acceso a los servicios básicos: agua, luz y transporte (Arauz, 2010).

Tipo de estudio.Este estudio es cualitativo, correspondiente a investigaciones de datos descriptivos, a partir de los cuales se desarrollan explicaciones y no se recogen los datos para validar hipótesis, permitiendo el acceso a la información buscada, al mundo de las y los entrevistados (Vásquez, 2010).

Selección de la población. La selección de productores se realizó en base al registro existente de productores atendidos por el proyecto MST, que establecieron los SPA: GBCM y GBAD en el año 2006 (período comprendido 2006-2008).

La población total fue de 32 pobladores que incluyeron a productores y sus cónyuges; localizados en cinco caseríos: Buena Vista, El Guayabo, Los Guácimos, El Borbollón y Las Mercedes, los que se encuentran ubicados en la microcuenca "Guayabo-San José”.

En total existen 10 productores que tienen establecido el sistema GBAD y 6 productores con GBCM y 16 mujeres que son las cónyuges de estos productores.

Procedimientos y métodos de recolección de la información. Para la realización de este estudio se creó un equipo de trabajo, el que involucró a un representante del proyecto MST, específicamente el coordinador del componente agroforestal, responsable de promover los 
dos SPA: GBCM y GBAD, investigadoras y un profesor investigadorde la UNAN-León.

La realización del trabajo inició con un acercamiento a la oficina del proyecto MST, para la revisión de la información existente.

Seguidamente se procedió al acceso al campo, ésta fase tuvo una duración de cuatro semanas, visitando en cada uno de los caseríos las casas y las parcelas de la población de estudio, recopilando así la información de interés.

La información se obtuvo a través de la triangulación de métodos: entrevistas, observación participante, y grupos focales.

Entrevista. Se realizó una entrevista piloto con una pequeña muestra de la población, para valorar su adecuación y posibles fuentes de error, una vez validada la entrevista se aplicó a la población en estudio, en la recolección de datos las investigadoras se encargaron de las entrevistas y sus respectivas grabaciones.

Las entrevistas se transcribieron, seleccionando la información de interés respecto a los objetivos planteados.

Posteriormente se realizaron entrevistas informales al Coordinador del proyecto MST y al Ex Director de la UNAG de El Sauce.

Observación participante. La observación participante se realizó con el fin de complementar la información y obtener datos acerca de las formas de vida y actividades que realizan la población en estudio.

Grupos focales. Se realizaron cuatro grupos focales, con los productores que tienen establecidos SPA y el equipo de investigación, dos en la escuela del caserío Las Mercedes y dos en la casa de uno de los productores del caserío El Guayabo.

Se presentó el tema y los objetivos de la investigación, estos tuvieron una duración de 45 y 60 minutos, un Docente investigador organizó y coordinó los eventos, una de las investigadoras desarrolló el papel de moderadora, dirigiendo al grupo a través de tópicos relacionados con los objetivos de la investigación (ver anexo, 5). La otra investigadora participó como observadora, anotando las expresiones del grupo y otros datos relevantes de su desenvolvimiento, complementando la información facilitada de manera individual en las entrevistas.

Concluido los grupos focales se procedió a transcribir la información generada por los productores, una vez transcrita y leídas se seleccionaron las expresiones textuales más importantes que permitían explicar el contexto de estudio.

Consideraciones éticas. Previo a la aplicación de los métodos de recolección de información, la población de estudio fue informada acerca de los objetivos de la investigación, se tomó en cuenta su consentimiento y se aseguró su confidencialidad en la información brindada, así como en las grabaciones realizadas.

Análisis. La información obtenida en los grupos focales y en las entrevistas se transcribió de forma textual, y posteriormente se realizó un análisis de contenido, que convierte los fenómenos registrados "en bruto" en datos que puedan ser tratados científicamente y construir con ellos un cuerpo de conocimientos.

Después de una lectura exhaustiva de las transcripciones e identificación de expresiones relacionadas con los objetivos, se analizó el contenido latente de las mismas (que es lo que quieren decir). La información es presentada en diagramas explicativos y citas textuales, para mejor la comprensión del estudio.

\section{RESULTADOS Y DISCUSIÓN}

Percepción de los productores sobre la aceptación de los sistemas productivos adaptativos. Las obras de conservación de suelo y agua son tecnologías promovidas por organismos públicos y privados preocupados por el deterioro de los recursos naturales. Algunas de estas prácticas se encuentran muy generalizadas en el ámbito agrícola del país, por lo que la difusión de tecnologías se realiza con el fin de aumentar la valoración económica, ecológica y social de los servicios ambientales.

En la microcuenca "Guayabo-San José" se han promovido tecnologías de CSA en las fincas de 16 productores, orientados a revertir la problemática ambiental e incrementar la producción agrícola de la zona, tomando en cuenta los procesos metodológicos, técnicos y culturales que intervienen en el proceso de aceptación de tecnologías, así como la percepción de los productores, encontramos una percepción positiva existiendo un 100\% de aceptación, considerando las siguientes razones expresadas por los productores:

Iniciativa por el proyecto "Manuel López". Los productores uno de los principales motivos de aceptación de los SPA, fue el desarrollo del proyecto "Manuel López" en los años 90; señalan que dicho proyecto fue uno de los mejores que se han desarrollado en la zona, representando el punto de partida para el cambio a una agricultura sostenible. El proyecto trabajó en la trasferencia de tecnologías de CSA, utilizando diferentes metodologías y medios de extensión agrícola, como la creación de promotores, establecimiento de parcelas demostrativas, técnicas de aprendizaje, brindaron además capacitaciones y asistencia técnica, las cuales eran dirigidas al establecimiento de áreas de conservación en las fincas de los productores atendidos por el proyecto; esto permitió que los productores lograran no solamente 
beneficios que podrían gozar por un período largo de tiempo, sino también que desarrollarán un sistema agrario local que les permitiera alcanzar mayores niveles de sostenibilidad ecológica y económica, lo que motivó el seguimiento de las tecnologías hasta la culminación del proyecto.

También es razonable lo que indica Fonseca (2006), en cuanto a la organización previa de una comunidad siendo este un proceso que ha logrado aumentar la participación de los agricultores permitiendo una mayor y mejor aceptación de tecnologías. Por esto se considera que el desarrollo del proyecto "Manuel López" marcó la pauta y estableció las bases para que los productores refirieran experiencias vividas que les permitiera trabajar posteriormente con otros proyectos que establecieran las mismas líneas de acción en el ámbito productivo. En base a lo anterior consideramos que uno de los motivos para lograr la aceptación del establecimiento de los SPA, desarrollados por proyecto MST fueron los resultados obtenidos con el proyecto "Manuel López".

Así lo expresa un productor con Sistema GBCM, del Caserío "Buena Vista" quien manifiesta que inició con el proyecto "Manuel López", continuó trabajando ya que se apropió de las prácticas, beneficios y resultados que iba obteniendo. Otro productor de Sistema GBAD, del mismo Caserío "Buena Vista" expresa entre otras cosas, que con el "Manuel López" conservaron el suelo, plantaron y cuidaron los árboles y que les ayudó a crear conciencia desde jóvenes de cultivar y cuidar los árboles, y cuidar el agua.

Nuevas tecnologías. Incrementar la productividad agrícola es un objetivo de cualquier proyecto de esta índole. Se inicia con la necesidad de enseñar innovaciones o nuevas tecnologías con el propósito de aumentar la productividad de la zona y por ende los rendimientos.

El cambio de las tecnologías es un aspecto normal de la conducta campesina, los productores continuamente están inmersos en procesos de cambios tecnológicos en sus unidades productivas. Si esta conducta de cambio no existiera, no se podrían adecuar de una manera más ajustada a los permanentes cambios ambientales, económicos, sociales y políticos que se producen tanto en el interior de sus sistemas, como en el entorno en el cual se desarrollan sus actividades productivas y en consecuencia, los sistemas campesinos no hubieran persistido por tanto tiempo. Ya que una agricultura que no cambia es o llega a ser a través del tiempo una agricultura poco productiva.

Uno de los informantes con Sistema GBCM, Caserío "El Guayabo" menciona "La idea es prosperar, dar respuesta a la alimentación y una respuesta más positiva a través del sacrificio del trabajo... la respuesta es diferente que cuando sembramos de forma tradicional, sin parcelear, sin darle un manejo correcto a la tierra, al final se viene trabajando menos y se produce más, en cambio cuando trabajamos así normal... toda la vida trabajamos, siempre sacrificados y no son muchas las ventajas o ganancias que se obtienen y eso es lo que me ha ido haciendo ir retomando un nuevo plan, un nuevo manejo de trabajo dentro de mi parcela"

Cáceres (1997), señala que la tecnología es un potencial para mejorar la producción agrícola, ya que no puede existir una sociedad desvinculada de la tecnología, considerando que quienes hacen la transferencia, deben tomar en cuenta las condiciones de vida de las familias y seleccionar aquellas que no rompan totalmente con su cultura productiva, sus hábitos y tradiciones agrícolas.

En base a esto el proyecto "MST" implementó modelos de sistemas productivos en las fincas de los productores de la microcuenca "Guayabo-San José", tomando en cuenta el potencial agroforestal de la tierra, los bajos rendimientos de producción, y el hecho de que en esta zona eran pocos los productores que estaban asociando sus cultivos con árboles y obras de conservación de suelos; a fin de que los productores incorporaran innovaciones tecnológicas que les permitiera adecuarse a los cambios y nuevas demandas que imponen los escenarios socioeconómicos emergentes. Un productor de Sistema GBCM, Caserío "Buena Vista", menciona que inició a trabajar con el sistema de callejones mejorados, ya que incluía árboles, granos básicos y otras especies.

Obtención de incentivos. Un factor considerable por parte de los productores para la aceptación de los SPA, son los incentivos obtenidos a través de las entidades promotoras de las tecnologías, dado que son pocos los productores que disponen de recursos económicos, financiamiento, herramientas y acceso a la asistencia técnica. Sin embargo existen estudios que demuestran altas tasas de aceptación en el momento que los productores son atendidos por un programa o entidad y que luego bajan drásticamente cuando dejan de recibir dichos beneficios ${ }^{1}$.

De acuerdo a esto muchas instituciones se han convencido, de que el uso de incentivos no solamente es inútil sino dañino y las razones son varias, cuando el progreso está acompañado de donaciones fácilmente se convence a la población de su incapacidad de progresar por su propio esfuerzo. De manera similar Toruño (2001) indica que el crédito para las actividades agroforestales constituyó el instrumento financiero para la implementación de los paquetes tecnológicos propuestos por el proyecto "Los Maribios". Uno de los entrevistados, productor con Sistema GBAD del Caserío "Las Mercedes" menciona que el proyecto los apoyó en la siembra de árboles, y que les proporcionaba semilla al inicio de la siembra, alambre para la reparación de cercos, etc.

Sin embargo se continúan implementando este tipo de proyectos debido a que se consiguen resultados más rápidos. Sin tomar en cuenta que la única forma de evitar el paternalismo es desarrollar proyectos capaces de fomentar un

\footnotetext{
${ }^{1}$ Cáceres, O. 2010. El rol de los Incentivos (entrevista). El Sauce.

Coordinador del proyecto MST. Comunicación personal.
} 
cambio de conducta e infundir entusiasmo en los productores.

Se debe crear una dinámica que pueda estimular el esfuerzo de verdadera auto-ayuda, lo que les permitirá aprender a planificar, encontrar soluciones a sus problemas, enseñar a otros, ganar confianza en sí mismo, desarrollar la creatividad e ingenio para mejorar su calidad de vida (CICA, 2003).

Abandono de los SPA. Un factor a considerar para el abandono de los sistemas es que el cambio en el sistema productivo ocurre a largo plazo, por lo que los beneficios obtenidos por estos no resuelven la situación actual a la que se enfrentan los productores, sumando a esto la ausencia de mano de obra disponible en el área rural debido a la emigración, que es uno de los principales problemas que afectan el desarrollo socioeconómico de la población rural.

La emigración de los productores de esta zona se presenta tanto a nivel nacional (a municipios como: Managua, León, Estelí y Chinandega), como a nivel internacional, encontrándose en este caso el principal país de destino: Costa Rica.

El motivo más predominante de este problema radica en los bajos ingresos económicos que perciben la mayoría de familias campesinas, debido a la instauración de modelos de producción tradicionales o convencionales que han demostrado ser insostenibles social, económica y ecológicamente.

Ante tal situación el gobierno está desarrollando prácticas tecnológicas que además de aumentar los ingresos de los productores les brinde un sistema de producción económico, ecológico y socialmente estable. Sin embargo mientras la población no cobre confianza en esas nuevas prácticas y en la labor de las instituciones de desarrollo agrícola estos no se podrán mostrar deseosos ni económicamente más capaces de intentar estas nuevas tecnologías y por otro lado el productor no estará en condiciones de sacar todo el partido a las prácticas que se deban emplear para mejorar su sistema productivo.

Presentándose tal escenario en 2 de los productores que aceptaron y luego abandonaron el SPA, como se muestra en la siguiente expresión de un padre de Productor con Sistema GBCM, Caserío "Los Guácimos": "Lo que pasa es que el sistema era de mi hijo... pero él lo dejó de trabajar porque no le daba con lo que sacaba en el sistema y por eso se fue para Costa Rica"

Percepción de los productores sobre la adopción de los SPA. Los factores relacionados con la adopción de nuevas tecnologías comprenden tanto las características personales como las características del marco en que actúan los productores, por lo que no todos adoptan una tecnología al mismo tiempo, por conveniente que pueda ser. Inclusive la mayoría de las nuevas tecnologías van y vienen sin gran importancia aunque algunas de ellas se pueden llegar a considerar con suficiente mérito por algunas personas como para que al menos la acepten y las experimenten.

El esfuerzo realizado para explicar el comportamiento adoptante ha tendido a centrar la atención en el carácter innovador como característica del individuo (CICA, 2003).

Por otra parte se puede considerar el hecho de que el productor arrendatario difícilmente es libre de tomar algunas decisiones, dado que no cuenta con la seguridad de que los beneficios sean para su desarrollo.

Tenencia de la tierra. La tenencia de la tierra define muchas veces qué tipo de tecnología tiene una posibilidad alta de ser adoptada. A como señala Toruño (2001) la tenencia de la tierra resulta ser un factor muy determinante en la adopción de los sistemas agroforestales. Las mayores probabilidades de adopción aumentan cuando los productores y productoras cuentan con terreno propio, ya que un agricultor arrendatario difícilmente adopta tecnologías con resultados a mediano y largo plazo (ej. barreras vivas, frutales, etc.).

Según estudios (Arauz, 2010), el 100\% de los productores en la microcuenca "Guayabo-San José”, poseen algún tipo de documento que los acreditan como propietarios de sus tierras. Como señala uno de los productores con Sistema GBCM, Caserío "Buena Vista": "La tierra es Propia, mejor dicho familiar porque nosotros no alquilamos..."

Considerando lo anterior, el proyecto tomó como uno de sus criterios de selección para el establecimiento de los sistemas, que los productores fueran dueños de sus tierras; ya que éstos presentan mayores probabilidades de adopción de tecnologías, porque tienen seguridad en la tierra que poseen, además que reconocen que sus propiedades adquieren un valor agregado con la introducción de los sistemas. De ahí la convicción de muchos productores (Grupo focal con productores que establecieron SPA en la microcuenca "Guayabo-San José") quienes valoran que con la introducción de buenas prácticas agrícolas la tierra adquiere mayor valor, y que conservándola podrán heredar a sus hijos el medio que les permitirá el sustento futuro.

Beneficios obtenidos. La implementación de estos sistemas productivos adaptativos, aumenta la productividad agrícola y por lo tanto, es un factor importante de combate a la pobreza, contribuyen al funcionamiento de los ecosistemas y protegen la diversidad biológica.

Como resultado de la implementación de estos sistemas, se mencionan algunos beneficios relacionados con el incremento de la producción, restauración de los ecosistemas, recuperación de la biodiversidad ante el avance de la deforestación, desertificación, producción sana en las parcelas productivas así como la reducción de la erosión del suelo y con ello la reducción de la contaminación de las aguas. 
Algunos de los beneficios referidos por los productores, es la conservación de suelo, mejorando de este modo la estructura y textura del mismo, produciendo un aumento en la fertilidad, reduciendo así los costos de producción por la poca utilización de insumos externos.

Los productores, como lo señala un productor con Sistema GBCM, Caserío "Las Mercedes", la práctica de conservación de suelo le permite que la tierra no esté totalmente desprotegida de las lluvias, evitan el truncamiento del suelo, se reduce la erosión, por tanto con las obras de conservación de suelo y la no quema permite conservar el suelo y permite la permanencia de la maleza lo que contribuye a la fertilidad del suelo.

También mencionan la obtención de productos extras por la diversificación de sus parcelas, lo que además de representar un aumento de la producción asegura un ingreso capaz de mejorar su economía, garantizando la seguridad alimentaria de sus familias, complementando una mejor actitud respecto a sus sistemas de producción. Como lo indica un Productor Sistema GBAD, Caserío "Buena Vista", "El gran beneficio que puedo obtener son mis árboles... y he podido tener mi comida también, porque la verdad de las cosas que yo pienso que no es para ahorita es para mañana, porque en el futuro mis hijos, van a necesitar esa madera, mis nietos y ... el beneficio no es tan grande ahorita pero si va a ser más grande más adelante, porque el volumen de producción va a creciendo."

Capacitación y asistencia técnica. Las capacitaciones se ocupan de orientar a las personas para que tomen decisiones de acuerdo a sus propios intereses y bienestar, además ofrece los elementos necesarios para mejorar su calidad de vida, la que acompañada de una apropiada asistencia técnica crean los elementos de sostenibilidad dirigido a evaluar la continuidad de los proyectos de desarrollo rural (CICA, 2003).

El rol de los agentes externos es de vital importancia en el proceso de adopción, ya que no sólo deben funcionar como un mediador que pone a disposición de los productores una nueva propuesta técnica, sino que también debe convertirse en un catalizador del proceso. Lejos de ser un proceso unidireccional, la incorporación de una nueva tecnología por parte de los productores implica un flujo activo de intercambio de información entre dos sujetos sociales esencialmente distintos.

La asistencia técnica y las capacitaciones son importantes para asegurar la implementación exitosa de nuevas tecnologías, lo cual es un pre requisito para su posterior adopción.

Tomando en cuenta lo expresado por los productores de la microcuenca, aquellos que no reciben asistencia técnica han representado bajos porcentaje de adopción, ya que existen tecnologías que demandan mayor apoyo para su orientación y seguimiento, (haciendo mención de algunas como: la construcción de diques, curvas a nivel, barreras vivas, barreras muertas, cercas vivas, no quema, rotación de cultivos, abonos verdes, etc.) la aplicación incorrecta de las nuevas tecnologías trae como consecuencia la no apreciación de los beneficios que se esperan obtener de su sistema de producción.

También incide en el proceso de adopción la coordinación interinstitucional, en una zona de impacto determinada. En muchos casos, la adopción de determinada tecnología se ve limitada cuando no existe ningún tipo de coordinación entre las entidades involucradas, siendo éste uno de los puntos débiles encontrados en evaluaciones realizadas al proyecto, coincidiendo con la opinión de los productores quienes mencionan desconocer la relación existente entre INTA y MST como instituciones involucradas en el proceso de desarrollo de los sistemas productivos.

De igual manera cuando los técnicos no se presentan en las comunidades, por una u otra razón, y no se cubre la plaza, quedando la zona sin atención el problema se agudiza, pues como señalan los productores ellos demandan además de capacitaciones, visitas y asistencia técnica de manera práctica y en la parcela, con mayor frecuencia y calidad de seguimiento.

Aumento del área de los sistemas productivos adaptativos. Para determinar la adopción de tecnologías de conservación de suelo y agua, se toma en cuenta el aumento del área en que se está aplicando las tecnologías en las fincas.

Uno de los criterios que consideró el MST para establecer los SPA en las fincas de los productores era que estos dispusieran de un área aproximada de $1.5 \mathrm{mz}$ para la aplicación de las tecnologías, la cual pudo haber aumentado en dependencia del porcentaje de adopción que los productores desarrollaran. Siendo este un factor determinante que lograra indicar si los productores adoptaron o no el nuevo modelo de producción.

A través de las entrevistas y grupos focales realizadas con los productores de la microcuenca "Guayabo-San José", encontramos que el aumento del área se dio en 4 de los 16 productores que representan la población de estudio. Lo que indica que la tecnología no fue adoptada en su totalidad debido a factores endógenos y exógenos que interfieren con las actividades del productor.

Manejo de los sistemas productivos adaptativos. El modelo es un sistema productivo agroforestal que combina tres componentes: Agrícola, Forestal y Obras de Conservación de Suelos y Agua, este modelo sugiere un manejo intensivo de la tierra, en el corto y mediano plazo, en función de cada uno de los componentes establecidos, para lograr un aumento sustancial de la producción (Manejo Sostenible de la Tierra et al., 2005).

Los productores de la microcuenca "Guayabo-San 
José", expresaron haber establecido sólo las prácticas que estaban a su alcance y no todas aquellas contempladas en el modelo del sistema, ya que éstas conllevan mucho trabajo, mano de obra, tiempo y recursos económicos y por eso algunos no las ponen en práctica. A como lo expresa un Productor con Sistema GBCM, Caserío "El Guayabo"), "No es un trabajo fácil, se necesita ponerle interés y amor, ese es el problema que se ha venido dando en toda la comunidad, hay gente que empieza con ganas, si hay dinero con facilidad se avanza más y cuando no hay que esforzarse más con la familia y los hijos que son los que te ayudan, entonces no es mucho lo que se hace y el tiempo que se lleva en conservar terreno con barreras y esas cosas es un tiempo que será mucho y que se hace poco".

Sin embargo aunque no establecieron todas las prácticas del modelo planteado por el proyecto, sus sistemas productivos. Las prácticas realizadas por los productores de la microcuenca "Guayabo-San José", en los tres componentes de los sistemas productivos adaptativos son : el componente agrícola es caracterizado por siembra al espeque, incorporación de rastrojo, Siembra e incorporación de abonos verdes y barreras vivas El componente forestal constituido por manejosilvícola e incorporación de rastrojos y el componente conservación de suelo y agua constituida por construcción de barreras muertas, construcción de diques y mantenimiento de las obras.

Esto concuerda con lo manifestado por Cáceres (2007), quien asegura que la mayoría de los pequeños productores difícilmente establecen nuevas tecnologías con propuestas muy estructuradas, debido a la elevada heterogeneidad en que se desarrollan sus actividades socio productivas, por lo que usualmente rescatan e incorporan sólo algunos de los elementos ofrecidos, transforman otros y, finalmente, ignoran los restantes componentes de la propuesta inicial.

Las prácticas establecidas por los productores dentro de sus sistemas productivos, requieren de un manejo adecuado el cual se ha ido modificando acorde a la disponibilidad de recursos y las características de sus sistemas. Como lo expresa un Productor con Sistema GBCM, Caserío "El Borbollón", quien manifiesta que en sus sistema tienen más barreras de la que le recomendaron, eso debido a la abundancia de piedras en su parcela lo que le permitió darse cuenta de, que se le facilitaba trabajar cuando no hay piedras, por lo que decidió quitarlas y hacer más barreras."

Los productores en la microcuenca "Guayabo- San José" señalaron realizar las siguientes prácticas de manejo en sus sistemas productivos:

Podas. Los productores expresan realizar esta actividad en las barreras vivas, árboles frutales y maderables dejando los residuos sobre el terreno para que se descompongan y se incorporen al suelo convirtiéndose en materia orgánica, facilitando así las actividades productivas en sus parcelas. Los productores (Grupo focal con productores que establecieron SPA en la microcuenca "El Guayabo-San José"), manifestaron entre otras cosas, que las podas de barreras se realizan cuando las plantas están crecidas, los residuos se dejan cubriendo el suelo para ser aprovechados como abono, y que a la hora de la siembra ya se han descompuesto facilitando la siembra. Otros manifiestan realizar la poda para dar forma a los árboles y aprovechar los residuos como abono

Manejo de sombra: Esta se realiza con el objetivo de reducir la competencia por luz solar entre los árboles y los cultivos establecidos en el sistema, para lograr un mejor desarrollo en los cultivos y por consiguiente una mayor producción. Los informantes mencionan (Productor con Sistema GBAD, Caserío "Las Mercedes"), que en la medida que los árboles producen sombra, se podan aquellos que tienen menor vigor, con el propósito de que se permita la entrada de luz al cultivo.

No quema. Los productores indican que al no quemar obtienen beneficios al medio ambiente aumentando la biodiversidad y mejorando la fertilidad de los suelos. De acuerdo a los informantes, la práctica de "no quema" permite que la maleza y los residuos de maíz quede en la superficie, lo que al descomponerse se constituyen en abonos orgánicos, la maleza que crece se chapea."

No introducción de animales dentro de las parcelas. Esta práctica se realiza para evitar la compactación de los suelos y la destrucción de las barreras. Los informantes mencionan que no introducen animales debido al costo que implica cada una de las obras y a lo fácil que resulta su destrucción por efecto del pisoteo de los animales quienes las destruyen.

Limpieza y mantenimiento de las obras de conservación. Los productores las realizan en cada ciclo de siembra, garantizando sus funciones como son: reducir la erosión, favorecer la infiltración y disminuir la escorrentía superficial de los suelos; señalan también realizarlas después del invierno siempre y cuando las lluvias hayan causado daño en las mismas. Los informantes manifiestan que año a año revisan el posicionamiento de las piedras si están en su logran, principalmente cuando las corrientes de las lluvias están atacando más fuerte. Por otro lado se da mantenimiento, si se derrumban las acomodan nuevamente, si hay derrumbes que ocasionan boquetes se socaban, o se afloja la tierra las vuelven a construir, limpian de maleza y las reconstruyen para la época seca.

Rol de las mujeres en el establecimiento y manejo de los sistemas productivos adaptativos. En base a la información obtenida con las cónyuges de los productores, consideramos 
que las mujeres no desempeñan funciones relevantes en las actividades destinadas a la producción agrícola, pues señalan que estas son labores propias de sus compañeros, dedicándose únicamente a las labores domésticas, a lo que las entidades no han hecho hincapié, ni le dan importancia al involucramiento de la mujer como un conjunto, sin tomar en cuenta que los beneficios indirectos que éstas reciben a través de sus cónyuges no es suficiente para lograr el grado de aceptación $\mathrm{y}$ adopción que los proyectos desean; y por consiguiente los niveles de adopción de las nuevas tecnologías aplicadas no son significativos.Una de las informantes expresa que su no involucramiento en el establecimiento y manejo de los SPA se debe a varios compromisos, donde destacan las tareas, incluyendo las domésticas como el cuido de los niños y atender la comida de trabajadores temporales en las labores agrícolas.

Hoy en día se reconoce que la mujer desempeña una función importante tanto en la producción agrícola como en el mercadeo, elaboración y preparación de alimentos; sin embargo aunque la mujer contribuye notablemente a la producción agrícola, raras veces su participación es considerada como un factor que influye en la sostenibilidad de los proyectos de desarrollo agrícola.

Se pueden alegar muchas razones para explicar por qué la mujer no se ha beneficiado directamente por estos proyectos, entre las que podemos mencionar: que no existen tecnologías para atender a las mujeres, en otros casos a pesar que existe la tecnología éstas están orientadas desatinadamente al hombre o las capacitaciones se realizan en horas y lugares no accesibles a la mujer, los cuales son obstáculos que deben superarse si se quiere que los países no vean limitada el uso de propuestas tecnológicas y aumenten su producción en conjunto con el desarrollo socioeconómico de sus familias.

\section{CONCLUSIONES}

En la implementación de los sistemas productivos adaptativos de la microcuenca "Guayabo-San José", impulsados por el proyecto MST, la perspectiva de los productores fue positiva, existiendo un 100\% (16 productores) de aceptación, considerando las siguientes razones: iniciativa por parte del proyecto "Manuel López", aplicación de nuevas tecnologías en sus sistemas y obtención de insumos.

Es considerable el abandono de dos de los sistemas productivos, debido a desconfianza de las nuevas tecnologías $\mathrm{y}$ a las condiciones socioeconómicas a las que se enfrentan los productores y sus familias, lo que ha traído como consecuencia la emigración.

El grado de adopción de los sistemas aumenta cuando los productores disponen de tierra propia, beneficios extras en sus parcelas y adecuadas capacitaciones y asistencia técnica. Sin embargo la adopción ha tendido a centrar la atención en el carácter innovador del individuo, por lo que aseveramos que la adopción de los sistemas se dio únicamente en cuatro de los 16 productores, tomando como factor de referencia el aumento del área donde han aplicado las nuevas tecnologías.

El manejo de los sistemas se encuentra acorde a la disponibilidad de recursos y las características de los sistemas de los productores. Por lo que no ponen en práctica todas aquellas contempladas en el modelo del sistema, sin embargo incluyen los tres componentes del modelo, señalando las siguientes: Poda, Manejo de sombra, No quema, No introducción de animales y limpieza y Mantenimiento de las obras.

Las entidades no tomaron en cuenta el involucramiento de las cónyuges de los productores en el proceso de adopción de los sistemas; a pesar que éstas pueden contribuir notablemente a la sostenibilidad y al cumplimiento de los objetivos del proyecto, considerando que desempeñan únicamente actividades domésticas y cuido de los hijos.

\section{REFERENCIAS BIBLIOGRÁFICAS}

Aráuz y Baquedano. 2007. Adopción de Tecnologías para la Producción de Frijol Rojo por Productores y Productoras de la microcuenca "Guayabo-San José", El Sauce, 2005-2007. Tesis de Máster en Desarrollo Rural Ecosostenible. El Sauce, Nicaragua. Universidad Politécnica de Nicaragua. $158 \mathrm{p}$.

Barzeb, R. 2004. Guía Práctica para la Evaluación Económica de Prácticas Productivas Sostenibles. Serie técnica \#13.Proyecto Establecimiento de un Programa para la Consolidación del Corredor Biológico Mesoamericano. 84 p.

Cáceres, D; Silvetti, F; Soto, G; Rebolledo, W. 2007. La adopción tecnológica en sistemas agropecuarios de pequeños productores. Agro sur v.25 n.2. 17 p.

CICA. 2003. Investigación para el desarrollo rural. Nuevas experiencias a XV años del CICA. I edición. México. 324 p.

FAO.1994. Memoria, Consulta de expertos sobre el avance de la agroforestería en zonas áridas y semiáridas de América Latina y El Caribe. Chile. 123 p.

Fonseca, Juan. 2006. Estudio sobre la adopción de variedades mejoradas de frijol en las principales zonas productoras de frijol de la región Brunca de Costa Rica. Agronomía Mesoamericana v. 17 n. 3. 357-367 p.

Montagnini, F.1992.Sistemas Agroforestales. Principios y aplicaciones en los trópicos. San José Costa Rica. 622 p.

MST/PNUD-MARENA. 2005. Modelo de granos básicos en callejones mejorados. Guía para implementación en zonas secas de Nicaragua. Managua. Nicaragua. $20 \mathrm{p}$.

MST/PNUD-MARENA. 2005 Modelo de granos básicos con árboles dispersos. Guía para implementación en zonas secas de Nicaragua. Managua. Nicaragua. 21p. 
OCÉANO, 2008. Enciclopedia Práctica de la Agricultura y la Ganadería. Barcelona, España. Editorial Océano. $753-754$ pp. Osorio, N. 2010. Descripción del Proyecto Manuel López. El Sauce, Nicaragua. Ex director de la UNAG. Comunicación personal. Ospina, A. 2006. Agroforestería. Aportes conceptuales, metodológicos y prácticos para el estudio agroforestal. Colombia. Editorial ACOSAC. 238 p.

Pichardo, V. 2007. Marco Conceptual de Género. (Diapositiva). Nicaragua. 15 diapositivas a color.

Romero, et al., 2005. Consultoría: Diagnóstico y propuestas para inversiones en el terreno sobre Sistemas Productivos y Prácticas de manejo sostenible de la tierra. Informe Final. Proyecto Manejo Sostenible de la Tierra. Nicaragua. 145 p.

Rugama, Rosa. 2005. Análisis de la situación de género en siete municipios de Nicaragua con áreas degradadas propensas a sequía. Informe final de consultoría de género. Nicaragua. $116 \mathrm{p}$.

Sagastume, Norman, et al., Guía para elaboración de estudios de adopción de tecnologías de Manejo sostenible de suelos y agua. Programa para la Agricultura Sostenible en Laderas de América Central PASOLAC. Documento No. 499 Serie Técnica 7/2006. 40 p. Toruño, Pedro. 2001. Adopción de sistemas agroforestales. Problemática y potencialidades para un manejo sostenible en la cordillera Los Maribios, Nicaragua. Tesis Mag. Sc. Georg-August Universitat Goettingen, RFA. 78 p. 2009. Principios básicos de la agroforestería en el trópico. Folleto. sl. León, Nicaragua.

UNICATS (2000). Manual para la Capacitación Agropecuaria. Agricultura Sostenible.

Vásquez, S. 2010a. Métodos y técnicas de investigación social, para indagar temáticas ambientales con comunidades o grupos sociales. (Diapositiva). Bagaces, Costa Rica. 10 diapositivas a color.

2010b. Técnicas Cualitativas para la Investigación Ambiental con comunidades o grupos sociales. (Diapositiva). Bagaces, Costa Rica. 16 diapositivas, color. 